DRAFT VERSION MAY 16, 2018

Preprint typeset using LTEX style emulateapj v. 5/2/11

\title{
LATE POP III STAR FORMATION DURING THE EPOCH OF REIONIZATION: RESULTS FROM THE RENAISSANCE SIMULATIONS
}

\author{
HaO XU ${ }^{1}$, Michael L. Norman ${ }^{1,2}$, BRian W. O'Shea ${ }^{3,4,5,6}$, AND JOHN H. Wise ${ }^{7}$ \\ ${ }^{1}$ San Diego Supercomputer Center, University of California, San Diego, 9500 Gilman Drive, La Jolla, CA 92093; hxu@ ucsd.edu, mlnorman@ucsd.edu \\ ${ }^{2}$ CASS, University of California, San Diego, 9500 Gilman Drive, La Jolla, CA 92093; \\ ${ }^{3}$ Department of Physics and Astronomy, Michigan State University, East Lansing, MI 48824, USA; oshea@msu.edu \\ ${ }^{4}$ Department of Computational Mathematics, Science and Engineering, Michigan State University, East Lansing, MI 48824, USA \\ ${ }^{5}$ National Superconducting Cyclotron Laboratory, Michigan State University, East Lansing, MI 48824, USA \\ ${ }^{6}$ JINA: Joint Institute for Nuclear Astrophysics and \\ ${ }^{7}$ Center for Relativistic Astrophysics, School of Physics, Georgia Institute of Technology, 837 State Street, Atlanta, GA 30332; jwise@ gatech.edu \\ Draft version May 16, 2018
}

\begin{abstract}
We present results on the formation of Pop III stars at redshift 7.6 from the Renaissance Simulations, a suite of extremely high-resolution and physics-rich radiation transport hydrodynamics cosmological adaptive-mesh refinement simulations of high redshift galaxy formation performed on the Blue Waters supercomputer. In a survey volume of about 220 comoving $\mathrm{Mpc}^{3}$, we found 14 Pop III galaxies with recent star formation. The surprisingly late formation of Pop III stars is possible due to two factors: (i) the metal enrichment process is local and slow, leaving plenty of pristine gas to exist in the vast volume; and (ii) strong Lyman-Werner radiation from vigorous metal-enriched star formation in early galaxies suppresses Pop III formation in ("not so") small primordial halos with mass less than $\sim 3 \times 10^{7} \mathrm{M}_{\odot}$. We quantify the properties of these Pop III galaxies and their Pop III star formation environments. We look for analogues to the recently discovered luminous Ly $\alpha$ emitter CR7 (Sobral et al. 2015), which has been interpreted as a Pop III star cluster within or near a metalenriched star forming galaxy. We find and discuss a system similar to this in some respects, however the Pop III star cluster is far less massive and luminous than CR7 is inferred to be.

Subject headings: galaxies: star formation - galaxies: high redshift - cosmology: early universe - methods: numerical - stars: Population III
\end{abstract}

\section{INTRODUCTION}

Although Population III stars (Pop III) have not been directly observed, they are thought to form from metal-free gas bound to dark matter minihalos since redshift $z \sim 20$ and are massive, having a large (tens to a few hundreds $\mathrm{M}_{\odot}$ ) characteristic mass (e.g. Abel et al. 2002; Bromm et al. 2002; O'Shea \& Norman 2007; Turk et al. 2009; Greif et al. 2012; Susa et al. 2014; Hirano et al. 2015). Due to their high mass, they have short lifetimes (Schaerer 2002) and may go supernovae ( $\mathrm{SNe}$ ), and enrich their surrounding intergalactic medium (IGM). Once the metallicity passes some critical metallicity, $\sim 10^{-6} \mathrm{Z}_{\odot}$ if dust cooling is efficient (Omukai et al. 2005; Schneider et al. 2006; Clark et al. 2008) or $10^{-3.5} \mathrm{Z}_{\odot}$ otherwise (Bromm et al. 2001; Smith et al. 2009), the gas can cool rapidly and lower its Jeans mass and form stars in clusters. These metal-enriched stars have a lower characteristic mass scale and most likely have an initial mass function (IMF) that resembles the present-day one.

Locally, the transition from Pop III to metal enriched star formation is solely dependent on the metal enrichment of future star forming halos from nearby Pop III and metal enriched star SN remnants. But globally Pop III star formation is also regulated by radiation feedback from all UV sources (Ahn et al. 2012). Lyman-Werner photons from earlier formed stars photodissociate $\mathrm{H}_{2}$ by the Solomon process and suppress the formation of Pop III stars in low-mass halos (Machacek et al. 2001; Wise \& Abel 2007; O'Shea \& Norman 2008; Xu et al. 2013), delaying Pop III star formation to occur in more massive halos (O'Shea \& Norman 2008; Xu et al. 2013). Metal enrichment is a local process (Whalen et al. 2008; Muratov et al. 2013; Wise et al. 2012a), involving complex interactions between SNe blastwaves, the intergalactic medium (IGM), halo mergers, and cosmological accretion. As a consequence of combining these two processes, Pop III stars may continue to form to low redshifts. This topic has been extensively studied with semi-analytic models (Scannapieco et al. 2003; Yoshida et al. 2004; Tumlinson 2006; Salvadori et al. 2007; Komiya et al. 2010), post-processing of numerical simulations (Karlsson et al. 2008; Trenti et al. 2009), and direct numerical simulations (Tornatore et al. 2007; Ricotti et al. 2008; Maio et al. 2010; Wise et al. 2012b; Muratov et al. 2013). For example, Trenti et al. (2009) suggested that Pop III stars may still form at the late epoch of $z=6$ in the under dense regions of the universe by post-processing of cosmological simulations with blast wave models. Muratov et al. (2013) also showed that Pop III stars continue to form until $z=6$ using direct cosmological simulations. But due to Pop III stars' short lifetimes (Schaerer 2002) and high metal enrichment effects (Nomoto et al. 2006; Heger \& Woosley 2002), the Pop III phase of a given galaxy should be very short $(\sim 10 \mathrm{Myr})$, making Pop III galaxies rare at any given redshift.

Recently Sobral et al. (2015) reported spectroscopic observations of the strong Lyman- $\alpha$ emitter CR7 at $z=6.6$ near the end of reionization. They interpreted the blue component and its associated nebular emission of CR7 as a massive Pop III star cluster with a nearby redder and more massive galaxy. This stimulated us to look for such objects in our Renaissance Simulations data (Xu et al. 2013; O'Shea et al. 2015). We report on the results of the formation of Pop III stars at low redshifts from the low density "Void" region of the Renaissance Simulations at $z=7.6$. Within the survey volume of $\sim 220$ 
comoving $\mathrm{Mpc}^{3}$, we found a total of 14 galaxies with young Pop III stars (formed within the past $\sim 10 \mathrm{Myr}$, and most of which are still alive at $z=7.6$ ). We first describe the model and simulation in Section 2. In Section 3, we present our results. We show the distribution of these Pop III galaxies, their properties and UV luminosities, and their star formation environment, including metallicity, Lyman-Werner background, and neighbor star forming galaxies. We identify a special case of a Pop III galaxy with a nearby large metal enriched star forming galaxy and discuss its similarities and differences to CR7. Finally, in Section 4 we examine why Pop III stars continue to form in our simulations at such low redshifts and discuss the implications of this work.

\section{THE RENAISSANCE SIMULATIONS}

The Renaissance Simulations were carried out using the ENZO $^{1}$ code (Bryan et al. 2014), which is an open source adaptive mesh refinement (AMR) code that has been extensively used for simulating cosmological structures, and in particular high-redshift structure formation (e.g., Abel et al. 2002; O'Shea \& Norman 2007; Turk et al. 2009; Wise et al. 2012a,b; Xu et al. 2013; Wise et al. 2014). Notably, UV ionizing radiation from Pop III and metal-enriched stellar populations is followed using the MORAY radiation transport solver (Wise \& Abel 2011). Lyman-Werner radiation from stellar sources is first calculated in the optically-thin limit. We then include the attenuation of LW photons by a grey opacity approximation, the "picket-fence" modulation factor, as a function of the comoving distance between the source and the observer (Ahn et al. 2009). The properties of baryons (hydrogen, helium, and metals from stellar feedback) are calculated using a 9-species primordial non-equilibrium chemistry and cooling network (Abel et al. 1997), supplemented by metaldependent cooling tables (Smith et al. 2009). Prescriptions for Population III and metal-enriched star formation and feedback are employed, using the same density and metallicity criteria $\left(<10^{-4} \mathrm{Z}_{\odot}\right)$ as Wise \& Abel (2008) and Wise et al. (2012a). Pop III stars are formed with a range of masses using a Kroupa-like IMF but with a characteristic mass of $40 \mathrm{M}_{\odot}$. For each Pop III star formed its UV hydrogen ionizing and LW photon luminosities and lifetimes are determined by the mass-dependent model from Schaerer (2002), and their fates and chemical yields are as described in Wise et al. (2012a).

We simulated a region of the universe that is $28.4 \mathrm{Mpc} / \mathrm{h}$ on a side (or $40 \mathrm{Mpc}$ with $h=0.71$ ) using the WMAP7 $\Lambda$ CDM+SZ+LENS best fit cosmology (Komatsu et al. 2011): $\Omega_{M}=0.266, \Omega_{\Lambda}=0.734, \Omega_{b}=0.0449, h=0.71, \sigma_{8}=0.81$, and $n=0.963$. Initial conditions were generated at $z=99$ using MUSIC (Hahn \& Abel 2011), and a low-resolution $\left(512^{3}\right.$ root grid) simulation was run to $z=6$ to find regions suitable for re-simulation. The simulation volume was then smoothed on a physical scale of 8 comoving $\mathrm{Mpc}$, and regions of high $\left(\langle\delta\rangle \equiv\langle\rho\rangle /\left(\Omega_{M} \rho_{C}\right)-1 \simeq 0.68\right)$, average $(\langle\delta\rangle \simeq 0.09)$, and low $(\langle\delta\rangle \simeq-0.26)$ mean density (at $z=15,12.5$, and 8 , respectively) were chosen for re-simulation. These subvolumes, hereafter designated the "Rare peak," "Normal," and "Void" regions, with comoving volumes of 133.6, 220.5, and 220.5 $\mathrm{Mpc}^{3}$, were resimulated with three additional static nested grids, resulting in an effective initial resolution of $4096^{3}$ grid cells and particles in the region of interest, translating to a dark matter mass resolution of $2.9 \times 10^{4} \mathrm{M}_{\odot}$ in the same re- gion. We allowed further refinement in the Lagrangian volume of the finest nested grid based on baryon or dark matter overdensity for up to 12 total levels of refinement, corresponding to a comoving resolution of $19 \mathrm{pc}$ in the finest cells. For more details about the calculations and scientific results about the Renaissance Simulations, see Xu et al. (2013, 2014), Chen et al. (2014), and O'Shea et al. (2015).

In this work, we focus on the Void simulation, which has the slowest star formation rates and has been evolved down to $z=7.6$ to study the late Pop III star formation at redshifts below 8 . We do not include a uniform metagalactic LW radiation background in this specific simulation, as the LW radiation from local sources dominates the photon budget in our refined region. This simulation was performed on the NCSA Blue Waters supercomputer. Data were processed and analyzed using $\mathrm{YT}^{2}$ (Turk et al. 2011) on Blue Waters, the Gordon system at SDSC, and the Wrangler system at TACC.

\section{RESULTS}

We define a Pop III galaxy as a halo containing at least one active Pop III star, which is a star particle whose age is less than the main sequence lifetime of a Pop III star of that mass. We treat galaxies which have both primordial and metal-enriched star formation going on at the same time as Pop III galaxies. If a Pop III galaxy is a satellite of a metalenriched galaxy, however, we do not consider its parent a Pop III galaxy. Active stars emit ionizing and LW radiation during their lifetimes. After that they are assumed to be non-radiating stellar remnants, which we still track using Enzo's $N$-body machinery. After analyzing the stellar contents of all of the halos in the survey volume, we find approximately 500 metalenriched galaxies in the Void simulation at $z=7.6$, whereas Pop III galaxies are very rare with only 14 such objects in the volume.

We illustrate the distribution of the Pop III galaxies within the survey volume in Figure 1, where we show densityweighted projections of baryon density, volume-weighted LW radiation intensity, and density-weighted metallicity, with the halo IDs of the Pop III galaxies superimposed. We list halo properties and star formation environments of these galaxies in three tables. Table 1 gives virial properties, metallicity, and LW intensity, as well as the number and mass of young (active) and old (remnant) Pop III stars in these Pop III galaxies at $z=7.6$. As Pop III forming halos are more massive in the presence of strong LW radiation (O'Shea \& Norman 2008), they are usually forming multiple Pop III stars at the same time. Some of them have more than 10 Pop III stars with total stellar mass of over one thousand $\mathrm{M}_{\odot}$. Table 2 lists for each Pop III galaxy their ionizing UV luminosities, as well as the properties of their nearest neighbor galaxies. To better show the Pop III star forming environment, we also list in Table 3 the properties of the progenitors of these Pop III galaxies at $z=7.7$, which is about 12.1 Myr earlier.

Pop III galaxies are located in both low and high density regions, and have neighbors with total stellar masses ranging from just $10^{3} \mathrm{M}_{\odot}$ to up to a few times $10^{7} \mathrm{M}_{\odot}$. At this time, Lyman-Werner radiation, mostly from metal-enriched stars, has penetrated into the low density volume and is strong everywhere ( $>0.01 \mathrm{~J}_{21}, \mathrm{~J}_{21}$ is radiation intensity in units of $10^{-21}$ erg s $\mathrm{s}^{-1} \mathrm{~cm}^{-2} \mathrm{~Hz}^{-1} \mathrm{Sr}^{-1}$ ), delaying the Pop III formation and increasing the halo mass threshold for Pop III formation to 
Table 1

Pop III Galaxies at $z=7.6$

\begin{tabular}{|c|c|c|c|c|c|c|c|c|c|c|c|c|}
\hline $\begin{array}{l}\text { Halo ID } \\
\text { (1) }\end{array}$ & $\begin{array}{c}\mathrm{R}_{\text {vir }}(\mathrm{kpc}) \\
\text { (2) }\end{array}$ & $\begin{array}{c}\mathrm{M}_{\mathrm{gas}}\left(\mathrm{M}_{\odot}\right) \\
\text { (3) }\end{array}$ & $\begin{array}{c}\mathrm{M}_{\mathrm{DM}}\left(\mathrm{M}_{\odot}\right) \\
(4)\end{array}$ & $\begin{array}{c}\mathrm{M}_{\mathrm{vir}}\left(\mathrm{M}_{\odot}\right) \\
(5)\end{array}$ & $\begin{array}{c}\mathrm{Z}\left(\mathrm{Z}_{\odot}, \mathrm{MW}\right) \\
(6)\end{array}$ & $\begin{array}{c}\mathrm{J}_{\mathrm{LW}}\left(\mathrm{J}_{21}, \mathrm{MW}\right) \\
(7)\end{array}$ & $\begin{array}{c}\mathrm{Z}\left(\mathrm{Z}_{\odot}, \mathrm{VW}\right) \\
(8)\end{array}$ & $\begin{array}{c}\mathrm{J}_{\mathrm{LW}}\left(\mathrm{J}_{21}, \mathrm{VW}\right) \\
(9)\end{array}$ & $\begin{array}{l}\mathrm{N}_{\text {old }} \\
(10)\end{array}$ & $\begin{array}{c}\mathrm{M}_{\text {old }}\left(\mathrm{M}_{\odot}\right) \\
(11)\end{array}$ & $\begin{array}{l}\mathrm{N}_{\text {young }} \\
\text { (12) }\end{array}$ & $\begin{array}{c}\mathrm{M}_{\text {young }}\left(\mathrm{M}_{\odot}\right) \\
\text { (13) }\end{array}$ \\
\hline 144 & 1.72 & $2.16 \mathrm{e} 7$ & $8.55 \mathrm{e} 7$ & $1.07 \mathrm{e} 8$ & $1.81 \mathrm{e}-2$ & 2.43 & $1.86 \mathrm{e}-1$ & 1.91 & 14 & 788 & 1 & 8.86 \\
\hline 359 & 1.46 & $1.05 \mathrm{e} 7$ & $5.73 \mathrm{e} 7$ & $6.78 \mathrm{e} 7$ & $3.74 \mathrm{e}-2$ & 0.691 & $7.07 \mathrm{e}-2$ & 0.226 & 3 & 67.2 & 4 & 61.1 \\
\hline 448 & 1.45 & $1.22 \mathrm{e} 7$ & $5.53 \mathrm{e} 7$ & $6.75 \mathrm{e} 7$ & $2.27 \mathrm{e}-4$ & 62.1 & $1.25 \mathrm{e}-3$ & 7.79 & 0 & 0.0 & 18 & $1.15 \mathrm{e} 3$ \\
\hline 528 & 1.27 & $8.82 \mathrm{e} 6$ & $3.71 \mathrm{e} 7$ & $4.59 \mathrm{e} 7$ & $7.52 \mathrm{e}-5$ & 11.1 & $1.91 \mathrm{e}-4$ & 11.3 & 0 & 0.0 & 1 & 181 \\
\hline 599 & 1.36 & $8.50 \mathrm{e} 6$ & $4.60 \mathrm{e} 7$ & $5.45 \mathrm{e} 7$ & $2.95 e-3$ & 18.4 & $9.14 \mathrm{e}-4$ & 2.46 & 11 & 616 & 1 & 15.0 \\
\hline 728 & 1.20 & $1.27 \mathrm{e} 5$ & $3.65 \mathrm{e} 7$ & $3.66 \mathrm{e} 7$ & $5.48 \mathrm{e}-1$ & 2.81 & 8.48 & 1.39 & 31 & $1.68 \mathrm{e} 3$ & 4 & 28.8 \\
\hline 740 & 1.17 & $2.87 \mathrm{e} 6$ & $3.09 \mathrm{e} 7$ & $3.37 \mathrm{e} 7$ & 1.913 & 2.02 & 15.6 & 1.42 & 5 & 192 & 2 & 52.4 \\
\hline 763 & 1.23 & $5.77 \mathrm{e} 6$ & $3.38 \mathrm{e} 7$ & $3.96 \mathrm{e} 7$ & $3.76 \mathrm{e}-18$ & 31.5 & $4.29 \mathrm{e}-18$ & 1.75 & 0 & 0.0 & 6 & 299 \\
\hline 823 & 1.19 & $2.25 \mathrm{e} 6$ & $3.34 \mathrm{e} 7$ & $3.56 \mathrm{e} 7$ & $2.41 \mathrm{e}-2$ & 0.282 & 2.16 & 0.450 & 14 & 358 & 2 & 17.0 \\
\hline 927 & 1.20 & $1.08 \mathrm{e} 7$ & $2.75 \mathrm{e} 7$ & $3.83 \mathrm{e} 7$ & $9.13 e-05$ & 32.7 & $1.85 \mathrm{e}-4$ & 4.13 & 0 & 0.0 & 7 & 335 \\
\hline 998 & 1.10 & $5.58 \mathrm{e} 6$ & $2.38 \mathrm{e} 7$ & $2.94 \mathrm{e} 7$ & $8.89 \mathrm{e}-3$ & 27.1 & $6.98 \mathrm{e}-4$ & 3.47 & 5 & 426 & 9 & 357 \\
\hline 1200 & 1.08 & $4.47 \mathrm{e} 6$ & $2.23 \mathrm{e} 7$ & $2.68 \mathrm{e} 7$ & $1.51 \mathrm{e}-4$ & 12.3 & $3.86 \mathrm{e}-7$ & 0.554 & 5 & 242 & 0 & 0.0 \\
\hline 1316 & 1.11 & $7.76 \mathrm{e} 6$ & $2.20 \mathrm{e} 7$ & $2.98 \mathrm{e} 7$ & $2.77 \mathrm{e}-4$ & 28.6 & $4.28 \mathrm{e}-4$ & 3.38 & 4 & 237 & 5 & 285 \\
\hline 1344 & 1.07 & $3.30 \mathrm{e} 6$ & $2.31 \mathrm{e} 7$ & $2.64 \mathrm{e} 7$ & $8.05 e-2$ & 3.70 & 1.46 & 2.12 & 9 & 399 & 0 & 0.0 \\
\hline
\end{tabular}

Notes: Column (1): Halo ID. Column (2): Virial radius in proper kpc. Column (3): Baryon mass of the galaxy. Column (4): Dark matter mass of the galaxy. Column (5): total mass of the galaxy. Column (6): Mass-weighted metallicity. Column (7): Mass-weighted Lyman-Werner intensity. Column (8): Volumeweighted metallicity. Column (9): Volume-weighted Lyman-Werner intensity. Column (10): Number of Pop III remnants. Column (11): Mass of Pop III remnants. Column (12): Number of active Pop III stars. Column (13): Mass of active Pop III stars.

Table 2

Luminosities of Pop III Galaxies and its neighbors at $z=7.6$

\begin{tabular}{cccccccc}
\hline $\begin{array}{c}\text { Halo ID } \\
(1)\end{array}$ & $\mathrm{L}_{\mathrm{UV}, \text { PopIIII }}(\mathrm{erg} / \mathrm{s})$ & $\begin{array}{c}\mathrm{L}_{\mathrm{UV}, \text { enriched }}(\mathrm{erg} / \mathrm{s}) \\
(2)\end{array}$ & $\begin{array}{c}\text { Neighbor ID } \\
(3)\end{array}$ & $\begin{array}{c}\mathrm{M}_{1600} \\
(5)\end{array}$ & $\begin{array}{c}\mathrm{M}_{\star}\left(\mathrm{M}_{\odot}\right) \\
(6)\end{array}$ & $\begin{array}{c}\mathrm{L}_{\mathrm{UV}, \text { neighbor }}(\mathrm{erg} / \mathrm{s}) \\
(7)\end{array}$ & $\begin{array}{c}1(\mathrm{kpc}) \\
(8)\end{array}$ \\
\hline 144 & $1.11 \mathrm{e} 37$ & $1.93 \mathrm{e} 39$ & 59 & -11.3 & $1.02 \mathrm{e} 5$ & $9.31 \mathrm{e} 39$ & 7.91 \\
359 & $3.65 \mathrm{e} 38$ & $4.08 \mathrm{e} 38$ & 555 & -2.74 & $1.66 \mathrm{e} 3$ & 0 & 17.8 \\
448 & $5.58 \mathrm{e} 40$ & 0 & 618 & -2.23 & $9.56 \mathrm{e} 2$ & 0 & 11.0 \\
528 & $1.24 \mathrm{e} 40$ & 0 & 1 & -18.0 & $6.65 \mathrm{e} 7$ & $7.21 \mathrm{e} 42$ & 11.1 \\
599 & $7.55 \mathrm{e} 37$ & $9.03 \mathrm{e} 39$ & 192 & -10.1 & $1.54 \mathrm{e} 5$ & 0 & 17.2 \\
728 & $2.33 \mathrm{e} 37$ & $5.37 \mathrm{e} 39$ & 385 & -5.58 & $2.40 \mathrm{e} 3$ & 0 & 10.4 \\
740 & $7.05 \mathrm{e} 38$ & $3.72 \mathrm{e} 39$ & 187 & -4.48 & $8.49 \mathrm{e} 3$ & 0 & 13.8 \\
763 & $9.88 \mathrm{e} 39$ & 0 & 238 & $\ldots$ & $1.09 \mathrm{e} 4$ & 0 & 85.6 \\
823 & $1.70 \mathrm{e} 37$ & $3.03 \mathrm{e} 39$ & 1408 & -2.69 & $1.56 \mathrm{e} 3$ & 0 & 19.8 \\
927 & $1.33 \mathrm{e} 40$ & 0 & 10 & -15.2 & $2.36 \mathrm{e} 7$ & $3.62 \mathrm{e} 41$ & 7.83 \\
998 & $9.85 \mathrm{e} 39$ & $1.44 \mathrm{e} 39$ & 280 & -8.60 & $2.37 \mathrm{e} 4$ & $1.56 \mathrm{e} 39$ & 13.2 \\
1200 & 0 & $8.06 \mathrm{e} 38$ & 923 & -3.19 & $1.68 \mathrm{e} 3$ & 0 & 14.6 \\
1316 & $1.17 \mathrm{e} 40$ & 0 & 50 & -15.04 & $1.40 \mathrm{e} 7$ & $2.61 \mathrm{e} 41$ & 15.5 \\
1344 & 0 & $5.68 \mathrm{e} 39$ & 513 & -2.47 & $7.76 \mathrm{e} 2$ & 0 & 11.7 \\
\hline
\end{tabular}

Notes: Column (1): Halo ID. Column (2): Ionization UV luminosity of Pop III stars. Column (3): Ionizing UV luminosity of metal enriched stars. Column (4): Halo ID of nearest neighbor. Columns (5): Magnitude of UV in 1600 angstrom. Column (6): Stellar (metal enriched) mass of nearest neighbor. Column (7): Ionization UV luminosity of nearest neighbor. Column (8): Distance to nearest galaxy in proper kpc. UV luminosities in Columns 2, 3 and 7 are calculated using the same formulae as implemented in the simulations as described in Wise et al. (2012b). $\mathbf{M}_{1600}$ in Column 5 as calculated using spectrum synthesis as described in O'Shea et al. (2015).

Table 3

Progenitors of Pop III Galaxies at $z=7.7$

\begin{tabular}{|c|c|c|c|c|c|c|c|c|c|c|c|c|c|}
\hline $\begin{array}{c}\mathrm{ID}(z=7.6) \\
(1)\end{array}$ & $\begin{array}{c}\mathrm{ID}(z=7.7) \\
(2)\end{array}$ & $\begin{array}{c}\mathrm{R}_{\mathrm{vir}}(\mathrm{kpc}) \\
\text { (3) }\end{array}$ & $\begin{array}{c}\mathrm{M}_{\mathrm{gas}}\left(\mathrm{M}_{\odot}\right) \\
(4)\end{array}$ & $\begin{array}{c}\mathrm{M}_{\mathrm{DM}}\left(\mathrm{M}_{\odot}\right) \\
(5)\end{array}$ & $\begin{array}{c}\mathrm{M}_{\mathrm{vir}}\left(\mathrm{M}_{\odot}\right) \\
(6)\end{array}$ & $\begin{array}{c}\mathrm{Z}\left(\mathrm{Z}_{\odot}, \mathrm{MW}\right) \\
\text { (7) }\end{array}$ & $\begin{array}{c}\mathrm{J}_{\mathrm{LW}}\left(\mathrm{J}_{21}, \mathrm{MW}\right) \\
(8)\end{array}$ & $\begin{array}{c}\mathrm{Z}\left(\mathrm{Z}_{\odot}, \mathrm{VW}\right) \\
(9)\end{array}$ & $\begin{array}{c}\mathrm{J}_{\mathrm{LW}}\left(\mathrm{J}_{21}, \mathrm{VW}\right) \\
(10)\end{array}$ & $\begin{array}{l}\mathrm{N}_{\text {old }} \\
(11)\end{array}$ & $\begin{array}{c}\mathrm{M}_{\text {old }}\left(\mathrm{M}_{\odot}\right) \\
(12)\end{array}$ & $\begin{array}{c}\mathrm{N}_{\text {young }} \\
\text { (13) }\end{array}$ & $\begin{array}{c}\mathrm{M}_{\text {young }}\left(\mathrm{M}_{\odot}\right) \\
\text { (14) }\end{array}$ \\
\hline 144 & 142 & 1.71 & $2.05 \mathrm{e} 7$ & $8.50 \mathrm{e} 7$ & $1.06 \mathrm{e} 8$ & $1.36 \mathrm{e}-4$ & 1.08 & $1.82 \mathrm{e}-4$ & 1.09 & 0 & 0 & 0 & 0 \\
\hline 359 & 355 & 1.40 & $9.87 \mathrm{e} 6$ & $5.36 \mathrm{e} 7$ & $6.34 \mathrm{e} 7$ & $1.88 \mathrm{e}-13$ & $8.47 \mathrm{e}-2$ & $1.62 \mathrm{e}-12$ & $8.47 \mathrm{e}-2$ & 0 & 0 & 0 & 0 \\
\hline 448 & 434 & 1.41 & $1.15 \mathrm{e} 7$ & $5.26 \mathrm{e} 7$ & $6.40 \mathrm{e} 7$ & $2.15 \mathrm{e}-4$ & $9.43 e-2$ & $1.50 \mathrm{e}-3$ & $9.42 \mathrm{e}-2$ & 0 & 0 & 0 & 0 \\
\hline 528 & 528 & 1.27 & $8.68 \mathrm{e} 6$ & $3.78 \mathrm{e} 7$ & $4.65 \mathrm{e} 7$ & $4.35 e-5$ & 7.49 & $1.06 \mathrm{e}-4$ & 7.55 & 0 & 0 & 0 & 0 \\
\hline 599 & 612 & 1.32 & $8.24 \mathrm{e} 6$ & $4.45 \mathrm{e} 7$ & $5.27 \mathrm{e} 7$ & $4.10 \mathrm{e}-18$ & 23.7 & $4.49 \mathrm{e}-18$ & 1.13 & 5 & 349 & 4 & 172 \\
\hline 728 & 740 & 1.24 & $5.71 \mathrm{e} 6$ & $3.64 \mathrm{e} 7$ & $4.21 \mathrm{e} 7$ & $6.49 \mathrm{e}-2$ & 65.9 & $1.75 \mathrm{e}-2$ & 20.3 & 3 & 178 & 32 & $2.10 \mathrm{e} 3$ \\
\hline 740 & 796 & 1.18 & $5.82 \mathrm{e} 6$ & $3.08 \mathrm{e} 7$ & $3.66 \mathrm{e} 7$ & $1.27 \mathrm{e}-4$ & 0.164 & $3.50 \mathrm{e}-3$ & 0.164 & 0 & 0 & 0 & 0 \\
\hline 763 & 783 & 1.11 & $5.05 \mathrm{e} 6$ & $3.00 \mathrm{e} 7$ & $3.50 \mathrm{e} 7$ & $3.79 \mathrm{e}-18$ & $6.44 \mathrm{e}-2$ & $4.24 \mathrm{e}-18$ & $6.41 \mathrm{e}-2$ & 0 & 0 & 0 & 0 \\
\hline 823 & 846 & 1.20 & $6.16 \mathrm{e} 6$ & $3.24 \mathrm{e} 7$ & $3.85 \mathrm{e} 7$ & $7.58 \mathrm{e}-3$ & 28.8 & $4.59 \mathrm{e}-5$ & 1.26 & 8 & 358 & 8 & 182 \\
\hline 927 & 1054 & 1.19 & $1.03 \mathrm{e} 7$ & $2.71 \mathrm{e} 7$ & $3.38 \mathrm{e} 7$ & $8.36 e-5$ & 1.67 & $1.61 \mathrm{e}-4$ & 1.67 & 0 & 0 & 0 & 0 \\
\hline 998 & 976 & 1.09 & $5.56 \mathrm{e} 6$ & $2.34 \mathrm{e} 7$ & $2.89 \mathrm{e} 7$ & $1.82 \mathrm{e}-4$ & $2.61 \mathrm{e}-1$ & $4.19 \mathrm{e}-4$ & 0.261 & 0 & 0 & 0 & 0 \\
\hline 1200 & 1207 & 1.05 & $4.37 \mathrm{e} 6$ & $2.18 \mathrm{e} 7$ & $2.62 \mathrm{e} 7$ & $3.78 \mathrm{e}-18$ & $7.18 \mathrm{e}-2$ & $4.13 e-18$ & $7.01 \mathrm{e}-2$ & 0 & 0 & 0 & 0 \\
\hline 1316 & 1272 & 1.09 & $7.46 \mathrm{e} 6$ & $2.14 \mathrm{e} 7$ & $2.89 \mathrm{e} 7$ & $2.70 \mathrm{e}-4$ & 0.483 & $4.17 \mathrm{e}-4$ & 0.481 & 0 & 0 & 0 & 0 \\
\hline 1344 & 1266 & 1.06 & $4.41 \mathrm{e} 6$ & $2.32 \mathrm{e} 7$ & $2.76 \mathrm{e} 7$ & $7.99 \mathrm{e}-4$ & 50.6 & $3.08 \mathrm{e}-4$ & 5.47 & 0 & 0 & 3 & 334 \\
\hline
\end{tabular}

Notes: Column (1): Halo ID at $z=7.6$. Column(2): Halo ID at $z=7.7$. Column (3): Virial radius in proper kpc. Column (4): Baryon mass of the galaxy. Column (5): Dark matter mass of the galaxy. Columns (6): Total mass of the galaxy. column (7): Mass-weighted metallicity. column (8): Mass-weighted Lyman-Werner intensity. Column (9): Volume-weighted metallicity. Column (10): Volume-weighted Lyman-Werner intensity. Column (11): Number of Pop III remnants. Column (12): Mass of Pop III remnants. Column (13): Number of active Pop III stars. Column (14): Mass of active Pop III stars. 

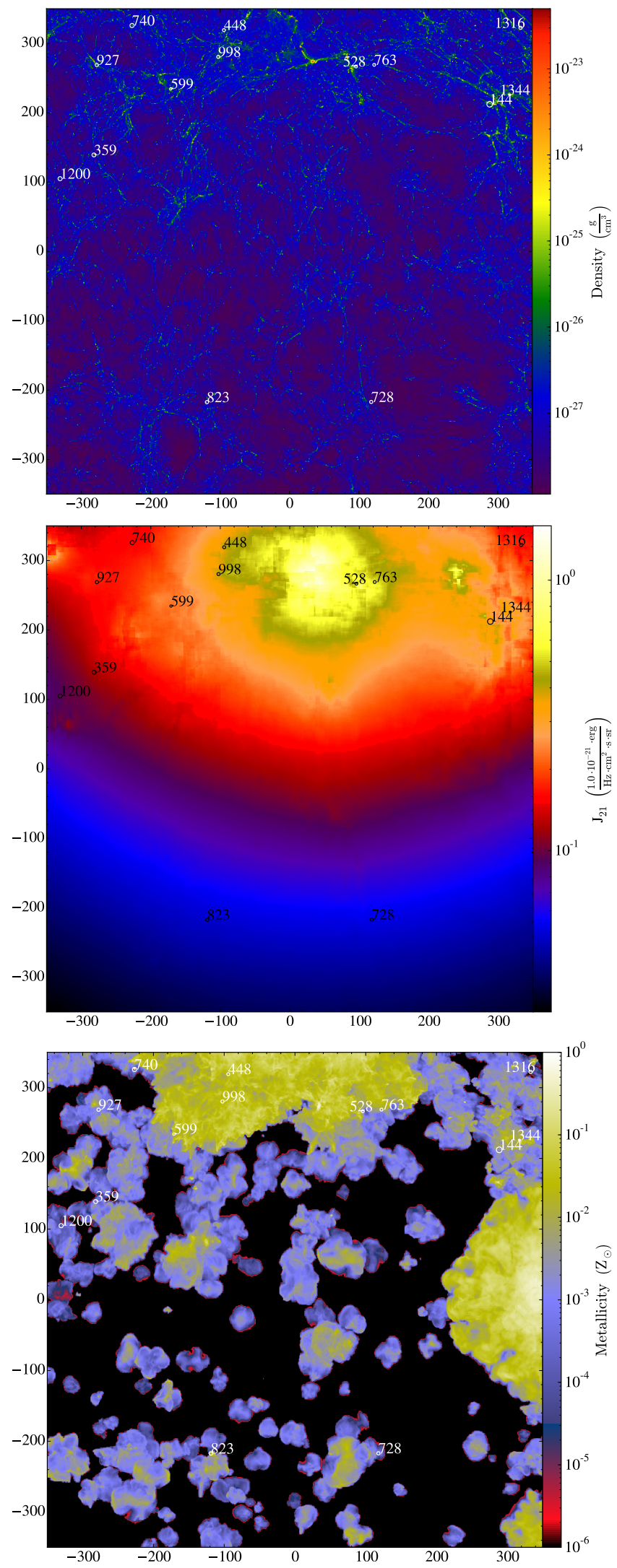

Figure 1. Projections through the Void survey volume of gas density (top), Lyman-Werner radiation intensity (middle), and metallicity (bottom) at $z=$ 7.6 with Pop III galaxies marked with their halo number. The survey volume is 6 comoving $\mathrm{Mpc}(\sim 700 \mathrm{kpc})$ on a side.
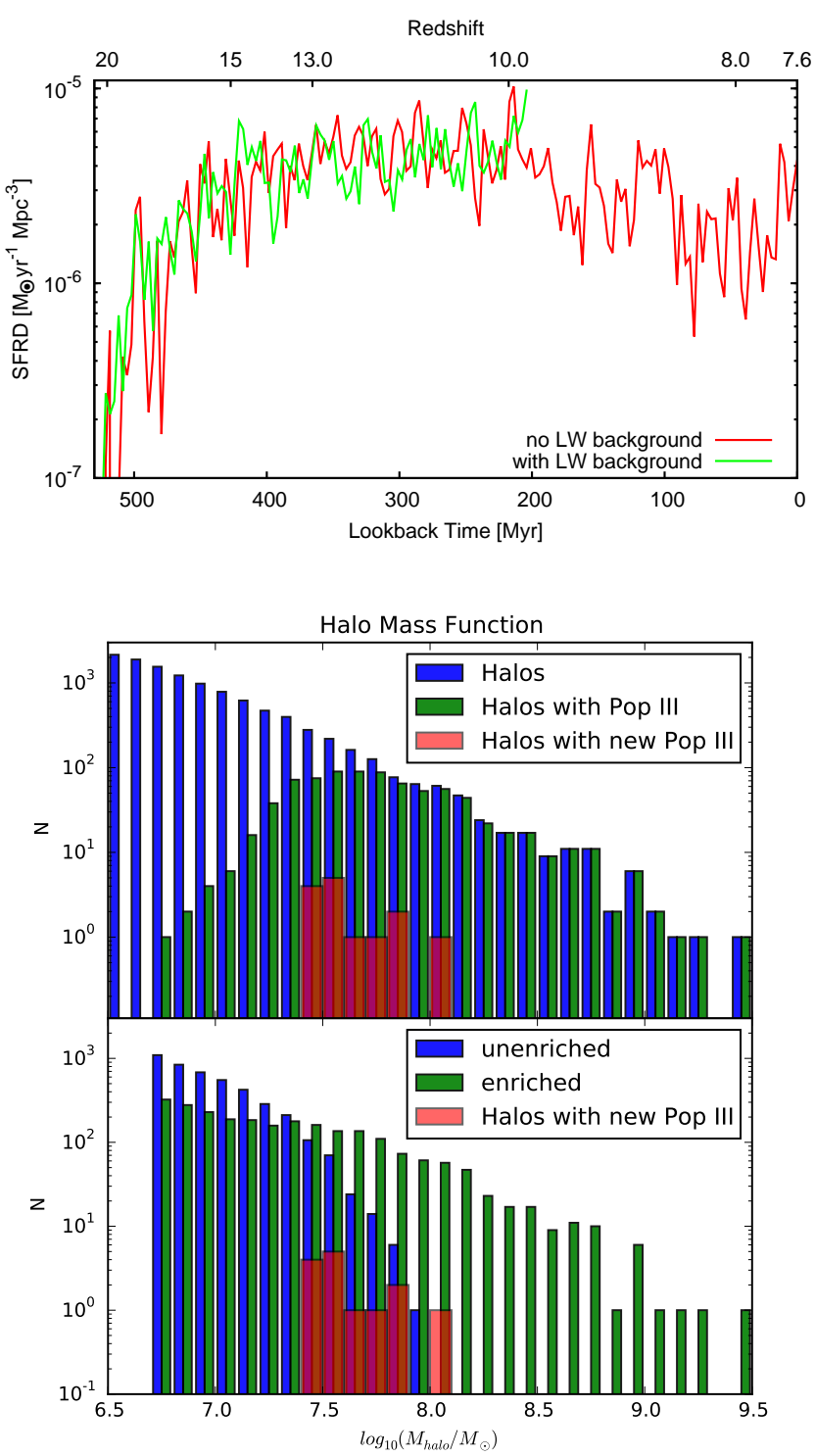

Figure 2. Top figure: Cosmic evolution of star formation rate density for Pop III. Red solid line shows the results from this simulation, and green dash line shows the results from the simulation of the same region with a uniform metagalactic Lyman-Werner background based on the average star formation rate density. Bottom figure: Halo mass function (non cumulative). Upper panel: Blue bins show all halos, and green bins show halos with Pop III stars and remnants; lower panel: Blue bins show metal enriched halos (volume weighted metallicity inside virial radius $[\mathrm{Z} / \mathrm{H}]>-4$ ), green bins show metal poor halos $([\mathrm{Z} / \mathrm{H}]<-4)$. In both panels, Red bins shows halos with recent Pop III star formation. The upper panel shows all halos found by HOP halo finder, while the lower panel shows virialized halos only. Sub-halos (halo inside a larger halo) are also removed from the bottom panel.

$\sim 3 \times 10^{7} \mathrm{M}_{\odot}\left(\mathrm{O}^{\prime}\right.$ Shea \& Norman 2008). By contrast, metal enrichment is local and much less efficient at affecting Pop III star formation globally (Whalen et al. 2008). Even inside metal-enriched stellar cluster forming regions, there are still halos with pristine gas able to form new Pop III stars.

The top panel of Figure 2 shows the redshift evolution of the Pop III star formation rate density. We also show the results from the simulation of the same region with a uniform metagalactic Lyman-Werner background based on the average star formation rate density (O'Shea et al. 2015) to $\mathrm{z} \sim 10$. LW background has little impact on the Pop III star formation rate density, since LW flux inside Pop III forming regions is 

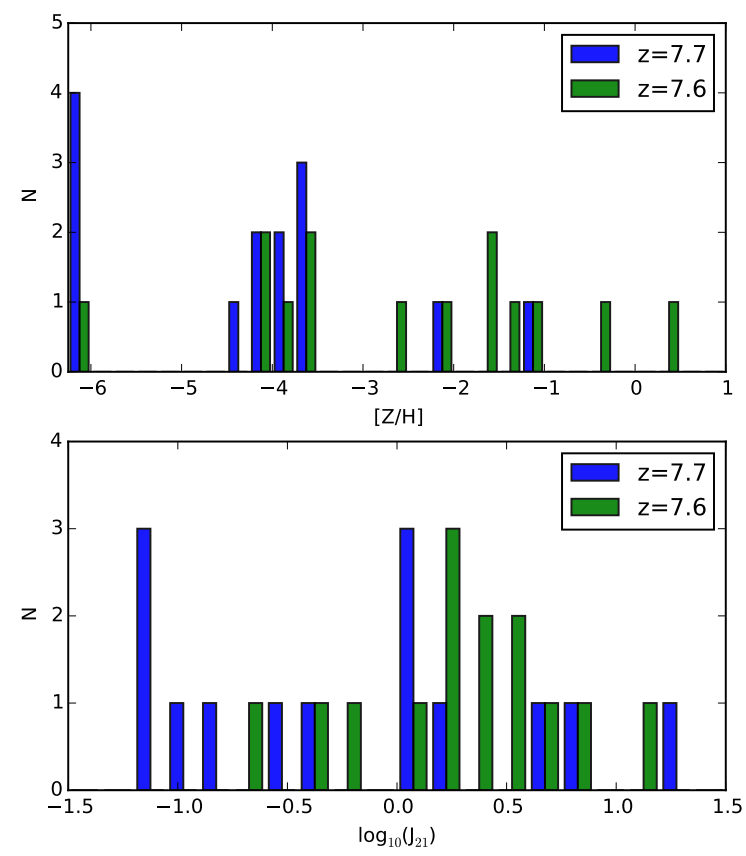

Figure 3. Mass-weighted averaged (inside virial radii) metallicity (top) and volume-weighted averaged Lyman-Werner intensity (bottom) distributions of the recent Pop III star forming galaxies. Green shows the halos at $z=7.6$, while blue shows their progenitors at $z=7.7$. The first bin in metallicity histogram shows the accumulcated stars with metallicity $[\mathrm{Z} / \mathrm{H}]<-6$.

dominated by local sources. More specifically, the LW background delays the Pop III formation for about $20 \mathrm{Myr}$ for $\mathrm{z}$ $=13-10$. At $\mathrm{z}=9.8$, there is about $7 \%$ percent more Pop III stellar mass in the no LW background simualtion. The SFR density grows gradually from redshifts larger than 20 to $\sim$ $10^{-5} \mathrm{M}_{\odot} \mathrm{yr}^{-1} \mathrm{Mpc}^{-3}$ at $z=10$ to 13 . There is a clear trend that the SFR density decreases slowly with decreasing redshift after $z=10$, but Pop III stars continue to form at a significant rate at $z<8$. The bottom panel of Figure 2 contains the halo mass functions showing halos with and without Pop III stars and their enrichment status. The Pop III galaxies concentrate in the range $M_{\text {vir }}=10^{7.5}-10^{8} \mathrm{M}_{\odot}$, as they just pass the threshold to form Pop III in a strong LW radiation environment.

Figure 3 shows the frequency of Pop III galaxies and their progenitors as a function of metallicity and local LymanWerner radiation intensity. While Pop III galaxy progenitors have low metallicity, their halos are quickly enriched when Pop III stars form and die as a supernova, and then complete the Pop III to metal-enriched star formation environment transition in less than $10 \mathrm{Myr}$. And due to their higher masses, Pop III galaxies are likely to keep their gas after Pop III supernova explode and they begin to form metal-enriched stars immediately. Out of 14 Pop III galaxies, there are 11 galaxies also forming metal-enriched stars. As the Lyman-Werner radiation intensity over all these halos is already at $0.1 \mathrm{~J}_{21}$ or more, LW radiation from the newly formed Pop III and metal-enriched stars has only mild impacts on the total LW intensity.

Figure 4 shows projections of gas density, metallicity, temperature, and Lyman-Werner flux of a metal-enriched galaxy with a stellar mass $M_{\star}=6.7 \times 10^{7} \mathrm{M}_{\odot}$ and a nearby halo (ID $\left.528 ; M_{\text {vir }}=4.6 \times 10^{7} \mathrm{M}_{\odot} ; M_{\text {gas }}=8.8 \times 10^{6} \mathrm{M}_{\odot}\right)$ that is 11.1 kpc away and has only formed a single Pop III star. The UV luminosity of the Pop III galaxy is only $1.2 \times 10^{40} \mathrm{erg} \mathrm{s}^{-1}$, or about $1 / 600^{\text {th }}$ of its metal-enriched galaxy neighbor. The feedback of the metal-enriched stars from the large galaxy neighbor has heated and enriched the gas surrounding the Pop III galaxy, but fails to change the pristine status of its core region, allowing the Pop III star to form. As the radiation ionizes the enriched medium surrounding the Pop III galaxy, metal nebular emission lines will be produced even though the ionizing source is metal-free.

We compare this simulated system with the inferred properties of CR7 that could be interpreted as a Pop III galaxy with a stellar mass $\sim 10^{7} \mathrm{M}_{\odot}$ with an evolved galaxy with a stellar mass of $\sim 10^{10} \mathrm{M}_{\odot}$ that is 5 (projected) kpc away (Sobral et al. 2015). The total Ly $\alpha$ luminosity of CR7 was measured to be $8.5 \times 10^{43} \mathrm{erg} \mathrm{s}^{-1}$, which is an order of magnitude brighter than the UV luminosity of the simulated galaxy, $7.2 \times 10^{42} \mathrm{erg} \mathrm{s}^{-1}\left(\mathrm{M}_{1600}=-18.0\right)$.

The proximity of the two systems is the only similarity between CR7 and the simulated galaxy pair. While our simulated system is a low-mass analog to the Pop III interpretation to CR7, it is unlikely that CR7 is powered by a Pop III-like stellar population because rapid chemical enrichment will terminate any subsequent Pop III star formation in Halo 528. Such enrichment will originate externally from metalrich outflows, as seen in the metallicity projection of Figure 4 , and internally from Pop III supernovae. For a halo to form $\sim 10^{7} \mathrm{M}_{\odot}$ of Pop III stars, it will need to remain chemically pristine and form all of its stars in one burst, avoiding internal enrichment. This scenario requires a nearby galaxy that provides the Lyman-Werner flux to suppress Pop III star formation, but the halo cannot be too close as it will be externally enriched. Our simulated system illustrates such a "Goldilocks" scenario with the pristine halo having a gas mass $M_{\text {gas }}=8.8 \times 10^{6} \mathrm{M}_{\odot}$. We do not expect any pristine halos to have a much higher gas mass, and thus it is nearly impossible to produce a Pop III galaxy with a stellar mass $\sim 10^{7} \mathrm{M}_{\odot}$ that would require all of the gas to be converted into stars. We conclude that the Pop III-like interpretation of CR7 is highly unlikely (Visbal et al. 2016), and its nature could be explained by either a direct collapse black hole (Pallottini et al. 2015; Agarwal et al. 2015; Hartwig et al. 2015; Smidt et al. 2016; Smith et al. 2016) or a young metal-poor galaxy. In the latter case, the very blue color of CR7 could be caused by a sight-line that is aligned with a photo-ionized and photo-heated channel in which ionizing photons are escaping into the nearby intergalactic medium and destroying any dust grains within this channel (cf. Vanzella et al. 2016, for a $z=3$ analog).

\section{SUMMARY AND DISCUSSION}

The key results of this work are as follows:

1. Pop III stars continue to form at significant rates down to $z=7.6$, the termination redshift of the simulation. Pop III stars can still form in pristine pockets of gas inside metal enriched star forming regions.

2. Halos hosting late Pop III star formation ("Pop III galaxies") are significantly more massive at lower redshift than at higher redshift, with virial masses between $5 \times 10^{7}$ and $1 \times 10^{8} \mathrm{M}_{\odot}$ at $z \simeq 7.5$. This is due to the strong LW radiation from metal enriched star forming galaxies in their vicinity. Suppression of Pop III star formation in lower mass halos $\left(M_{\mathrm{vir}}<10^{7} M_{\odot}\right)$ extends the Pop III era. 

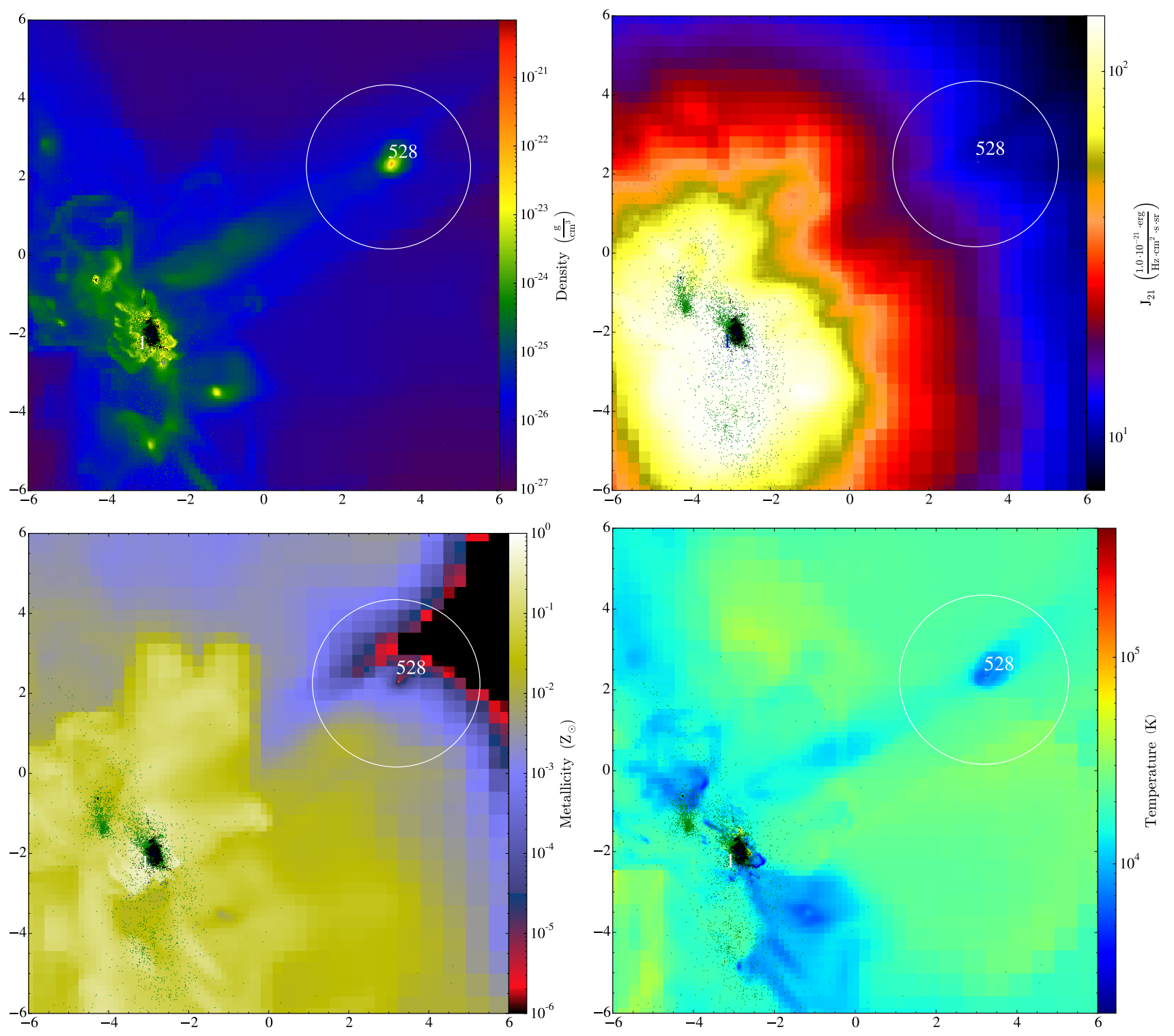

Figure 4. Projections of (from the top left panel, counter-clockwise) gas density, metallicity, temperature and Lyman-Werner radiation intensity of a volume including Halo 528 with a single young Pop III star and its metal enriched star forming galaxy neighbor. The projection volume is a cube of a side of 12 kpc. White dot shows the active Pop III star in Halo 528. Green dots are old metal enriched star clusters, while black dots are UV active metal enriched star clusters.

3. Pop III galaxies form multiple Pop III stars and also contain Pop III stellar remnants. A single Pop III galaxy could have more than 10 active Pop III stars and as much as $10^{3} \mathrm{M}_{\odot}$ in metal-free stellar mass.

4. One of our simulated Pop III and metal-enriched galaxy pairs is a low-mass analog of the Pop III-like interpretation of the observed CR7 galaxy (Sobral et al. 2015). However, we rule out this scenario as metal enrichment will quickly halt any further metal-free star formation after the initial burst.

Late formation of Pop III stars is caused by the combined effects of slow local metal enrichment and strong global LW radiation. While the metal-enriched bubbles have radii of tens of kpc at most, Lyman-Werner radiation from clustered metal enriched star galaxies can travel tens of Mpc deep into low density regions. Lyman-Werner radiation increases the halo mass threshold for Pop III formation (O'Shea \& Norman 2008), and furthermore in these larger halos, mixing timescales are longer for the metal-rich blastwave to advect from the virial radius to the star forming regions (as described in some detail in Smith et al. 2015). Therefore, Population III star formation is further delayed until the halo grows through slow cosmological accretion.

The formation of Pop III stars likely continues to even lower redshifts in this simulation. In the survey volume at $z=7.6$, only $6.22 \%$ of the volume and $13.01 \%$ of the gas mass is enriched to $[\mathrm{Z} / \mathrm{H}]>-4$. The entire volume with lower metallicity gas is immersed in strong LW radiation from metal enriched star forming galaxies. The fraction of the volume with Lyman-Werner intensity stronger than $1,0.1$, and $0.01 \mathrm{~J}_{21}$, is $5.12 \%, 55.37 \%$ and $100 \%$, respectively. The metal enrichment rate is even lower than the ionization rate. At this red- 
shift, there is only $16.1 \%$ of the volume or $17.7 \%$ of the mass of hydrogen that has been ionized. It is reasonable to expect that Pop III star formation will happen even after reionization is complete..

The survey volume less than 300 coming $\mathrm{Mpc}^{3}$ is very small, so any results from this simulation of late Pop III formation and Pop III galaxies should be considered as the upper limit on redshfits. Our simulation (with 0.062 Pop III galaxies per comoving $\mathrm{Mpc}^{3}$ at $\mathrm{z}=7.6$ ) suggests that Pop III galaxies are not rare at $\mathrm{z}=7-8$, at least in under dense regions. And out of these simulated 14 Pop III galaxies, there is one in a Pop III - metal enriched galaxies pair resembling CR7 in spatial distribution. Although the sample size is too small to draw any statistical significant conclusions, it is reasonable to expect there are many close Pop III - metal enriched galaxies pairs formed at this or even lower redshifts in the vast low density regions. Some of them may have Pop III stellar mass upto thousands $\mathbf{M}_{\odot}$ as other Pop III galaxies found in our simulations and could be much easier to be deteched.

We do not include a uniform metagalactic Lyman-Werner background based on the average star formation rate density; we only include Lyman-Werner flux from star formation in the refined region of our simulation. However we expect that the inclusion of LW background would not change our results significantly. This is because any additional LW radiation would only increase the halo mass threshold for Pop III formation and hence further delay their formation. These effects are weak when the LW intensity is higher than $J_{21}>0.1$ (O'Shea \& Norman 2008), which has been reached by local sources only in our present simulation. To check this hypothesis, we have performed the Void simulation with a LW background derived from the Normal region simulation, stopping at $z=10$. We find that this simulation with an uniform LW background has a very similar Pop III star formation history as the one reported on in this paper, as shown in the top panel of Figure 2.

The primary results of this paper - that Pop III star formation extends to late stages of reionization, and that Pop III stars form in clusters in higher mass halos - are significant for observations and our understanding of the formation of the first generation stars in the universe. It is beyond the sensitivity of JWST to directly see individual Pop III stars forming at high redshifts of $z=15-20$, except possibly their death through PISN explosions (Wise \& Abel 2005; Trenti et al. 2009; Hummel et al. 2012; Whalen et al. 2013). However, it could be much easier to discover and study Pop III clusters in a post-reionization environment as their formation is not halted during the early stages of reionization.

One possible important consequence of late Pop III star formation is that they might become strong $\mathrm{X}$-ray sources if their stellar remnants are in binary systems (Xu et al. 2014) with impacts on the X-ray background. X-rays photo-heat and preionize the IGM homogeneously on tens to hundreds of Mpc scales, and may be important to understand results of Ly $\alpha$ forest spectra (Tytler et al. 2009) and 21-cm signatures (Ahn et al. 2015). A detailed analysis of the X-ray background from Pop III binaries from the very beginning of Pop III stars $(z \sim 25)$ to the end of the reionization will be reported in a separate paper.

This research is part of the Blue Waters project, which a joint effort supported by the NSF (award number ACI1238993) and the state of Illinois, using NSF PRAC OCI-
0832662. This research was supported by NSF grants PHY0941373, AST-1109243, AST-1211626, and AST-1333360, and NASA grants NNX12AC98G, HST-AR-13261.01-A and HST-AR-13895.001. This work was performed using the open-source ENZO and YT codes, which are the products of collaborative efforts of many independent scientists from institutions around the world. Their commitment to open science has helped make this work possible.

\section{REFERENCES}

Abel, T., Anninos, P., Zhang, Y., \& Norman, M. L. 1997, New Astronomy, 2,181

Abel, T., Bryan, G. L., \& Norman, M. L. 2002, Science, 295, 93

Agarwal, B., Johnson, J. L., Zackrisson, E., Labbe, I., van den Bosch, F. C. Natarajan, P., \& Khochfar, S. 2015, ArXiv e-prints (1510.01733)

Ahn, K., Iliev, I. T., Shapiro, P. R., Mellema, G., Koda, J., \& Mao, Y. 2012, ApJL, 756, L16

Ahn, K., Shapiro, P. R., Iliev, I. T., Mellema, G., \& Pen, U.-L. 2009, ApJ, 695, 1430

Ahn, K., Xu, H., Norman, M. L., Alvarez, M. A., \& Wise, J. H. 2015, ApJ, 802,8

Bromm, V., Coppi, P. S., \& Larson, R. B. 2002, ApJ, 564, 23

Bromm, V., Ferrara, A., Coppi, P. S., \& Larson, R. B. 2001, MNRAS, 328, 969

Bryan, G. L., et al. 2014, ApJS, 211, 19

Chen, P., Wise, J. H., Norman, M. L., Xu, H., \& O’Shea, B. W. 2014, ApJ, 795,144

Clark, P. C., Glover, S. C. O., \& Klessen, R. S. 2008, ApJ, 672, 757

Greif, T. H., Bromm, V., Clark, P. C., Glover, S. C. O., Smith, R. J., Klessen, R. S., Yoshida, N., \& Springel, V. 2012, MNRAS, 424, 399

Hahn, O., \& Abel, T. 2011, MNRAS, 415, 2101

Hartwig, T., et al. 2015, ArXiv e-prints (1512.01111)

Heger, A., \& Woosley, S. E. 2002, ApJ, 567, 532

Hirano, S., Hosokawa, T., Yoshida, N., Omukai, K., \& Yorke, H. W. 2015, MNRAS, 448, 568

Hummel, J. A., Pawlik, A. H., Milosavljević, M., \& Bromm, V. 2012, ApJ, 755,72

Karlsson, T., Johnson, J. L., \& Bromm, V. 2008, ApJ, 679, 6

Komatsu, E., et al. 2011, ApJS, 192, 18

Komiya, Y., Habe, A., Suda, T., \& Fujimoto, M. Y. 2010, ApJ, 717, 542

Machacek, M. E., Bryan, G. L., \& Abel, T. 2001, ApJ, 548, 509

Maio, U., Ciardi, B., Dolag, K., Tornatore, L., \& Khochfar, S. 2010, MNRAS, 407, 1003

Muratov, A. L., Gnedin, O. Y., Gnedin, N. Y., \& Zemp, M. 2013, ApJ, 773, 19

Nomoto, K., Tominaga, N., Umeda, H., Kobayashi, C., \& Maeda, K. 2006, Nuclear Physics A, 777, 424

Omukai, K., Tsuribe, T., Schneider, R., \& Ferrara, A. 2005, ApJ, 626, 627

O'Shea, B. W., \& Norman, M. L. 2007, ApJ, 654, 66

-. 2008, ApJ, 673, 14

O’Shea, B. W., Wise, J. H., Xu, H., \& Norman, M. L. 2015, ApJL, 807, L12

Pallottini, A., et al. 2015, MNRAS, 453, 2465

Ricotti, M., Gnedin, N. Y., \& Shull, J. M. 2008, ApJ, 685, 21

Salvadori, S., Schneider, R., \& Ferrara, A. 2007, MNRAS, 381, 647

Scannapieco, E., Schneider, R., \& Ferrara, A. 2003, ApJ, 589, 35

Schaerer, D. 2002, A\&A, 382, 28

Schneider, R., Omukai, K., Inoue, A. K., \& Ferrara, A. 2006, MNRAS, 369, 1437

Smidt, J., Wiggins, B. K., \& Johnson, J. L. 2016, ArXiv e-prints (1603.00888)

Smith, A., Bromm, V., \& Loeb, A. 2016, ArXiv e-prints (1602.07639)

Smith, B. D., Turk, M. J., Sigurdsson, S., O’Shea, B. W., \& Norman, M. L. 2009, ApJ, 691, 441

Smith, B. D., Wise, J. H., O’Shea, B. W., Norman, M. L., \& Khochfar, S. 2015, MNRAS, 452, 2822

Sobral, D., Matthee, J., Darvish, B., Schaerer, D., Mobasher, B., Röttgering, H. J. A., Santos, S., \& Hemmati, S. 2015, ApJ, 808, 139

Susa, H., Hasegawa, K., \& Tominaga, N. 2014, ArXiv e-prints

Tornatore, L., Ferrara, A., \& Schneider, R. 2007, MNRAS, 382, 945

Trenti, M., Stiavelli, M., \& Shull, J. M. 2009, ApJ, 700, 1672

Tumlinson, J. 2006, ApJ, 641, 1

Turk, M. J., Abel, T., \& O'Shea, B. 2009, Science, 325, 601

Turk, M. J., Smith, B. D., Oishi, J. S., Skory, S., Skillman, S. W., Abel, T., \& Norman, M. L. 2011, ApJS, 192, 9

Tytler, D., Paschos, P., Kirkman, D., Norman, M. L., \& Jena, T. 2009, MNRAS, 393, 723

Vanzella, E., et al. 2016, ArXiv e-prints (1603.01616)

Visbal, E., Haiman, Z., \& Bryan, G. L. 2016, ArXiv e-prints (1602.04843)

Whalen, D., O'Shea, B. W., Smidt, J., \& Norman, M. L. 2008, ApJ, 679, 925

Whalen, D. J., Fryer, C. L., Holz, D. E., Heger, A., Woosley, S. E., Stiavelli,

M., Even, W., \& Frey, L. H. 2013, ApJL, 762, L6

Wise, J. H., \& Abel, T. 2005, ApJ, 629, 615 
H. Xu et al.

-. 2007, ApJ, 671, 1559

-.2008, ApJ, 685,40

-. 2011, MNRAS, 414, 3458

Wise, J. H., Abel, T., Turk, M. J., Norman, M. L., \& Smith, B. D. 2012a, MNRAS, 427, 311
Wise, J. H., Demchenko, V. G., Halicek, M. T., Norman, M. L., Turk, M. J., Abel, T., \& Smith, B. D. 2014, MNRAS, 442, 2560

Wise, J. H., Turk, M. J., Norman, M. L., \& Abel, T. 2012b, ApJ, 745, 50

Xu, H., Ahn, K., Wise, J. H., Norman, M. L., \& O'Shea, B. W. 2014, ApJ, 791,110

Xu, H., Wise, J. H., \& Norman, M. L. 2013, ApJ, 773,83

Yoshida, N., Bromm, V., \& Hernquist, L. 2004, ApJ, 605, 579 